\title{
Beta-blockers: friend or foe in asthma?
}

This article was published in the following Dove Press journal:

International Journal of General Medicine

5 July 2013

Number of times this article has been viewed

\section{Bente Arboe Charlotte Suppli Ulrik \\ Department of Pulmonary Medicine, Hvidovre Hospital and University of Copenhagen, Hvidovre, Denmark}

Correspondence: Charlotte Suppli Ulrik Department of Pulmonary Medicine 253, Hvidovre Hospital and University of Copenhagen, DK-2650 Hvidovre, Denmark

Tel +453862 6089

Fax +45 4583 6331

Email csulrik@dadlnet.dk
Background and aim: Recently, $\beta$-blockers have been suggested as a potential maintenance treatment option for asthma. The aim of this review is to provide an overview of the current knowledge of the potential benefits and risks of $\beta$-blocker therapy for asthma.

Method: Systematic literature review.

Results: No significant increase in the number of patients requiring rescue oral corticosteroid for an exacerbation of asthma has been observed after initiation of $\beta$-blocker treatment. Patients with mild to moderate reactive airway disease, probably both asthma and chronic obstructive pulmonary disease, may have a limited fall in forced expiratory volume in 1 second $\left(\mathrm{FEV}_{1}\right)$ following single-dose administration of $\beta$-blocker, whereas no change in $\mathrm{FEV}_{1}$ has been reported following long-term administration. In a murine model of asthma, long-term administration of $\beta$-blockers resulted in a decrease in airway hyperresponsiveness, suggesting an anti-inflammatory effect. In keeping with this, long-term administration of a nonselective $\beta$-blocker to steroid-naïve asthma patients has shown a dose-dependent improvement in airway hyperresponsiveness, and either an asymptomatic fall in $\mathrm{FEV}_{1}$ or no significant change in $\mathrm{FEV}_{1}$. Furthermore, available studies show that bronchoconstriction induced by inhaled methacholine is reversed by salbutamol in patients on regular therapy with a $\beta$-blocker. On the other hand, a recent placebo-controlled trial of propranolol and tiotropium bromide added to inhaled corticosteroids revealed no effect on airway hyperresponsiveness and a small, not statistically significant, fall in FEV in patients classified as having mild to moderate asthma.

Conclusion: The available, although limited, evidence suggests that a dose-escalating model of $\beta$-blocker therapy to patients with asthma is well tolerated, does not induce acute bronchoconstriction, and, not least, may have beneficial effects on airway inflammation and airway hyperresponsiveness in some patients with asthma. Further studies addressing the potential role of $\beta$-blocker therapy for asthma are clearly needed, but careful selection of the target population is warranted.

Keywords: asthma, beta-blockers, lung function, airway responsiveness

\section{Introduction}

Short-acting $\beta_{2}$-agonists (SABA) have been recommended for acute relief of asthma symptoms for decades, ${ }^{1}$ and long-acting $\beta_{2}$-agonists (LABA) are used as add-ons to inhaled corticosteroids (ICS) for patients not achieving asthma control on low-dose therapy with inhaled corticosteroids. ${ }^{1} \beta_{2}$-agonists are hence, together with ICS, the most commonly used drugs in the pharmacological management of asthma. ${ }^{1}$

It has been reported that chronic use of both SABA and LABA in asthma is associated with development of tolerance, an increase in airway hyperresponsiveness, poor asthma control, and even an increase in asthma mortality. ${ }^{2,3}$ Postmarketing studies 
were therefore initiated in order to clarify the safety of using LABA as maintenance therapy for patients with asthma., Although the Salmeterol Multicenter Asthma Research Trial ${ }^{4}$ and the Serevent Nationwide Surveillance Study ${ }^{5}$ have been much debated, not least with regard to the safety issue, they have added to the current recommendation that LABA should only be used as add-on therapy for patients not achieving disease control on ICS as monotherapy. ${ }^{1}$ However, on the basis of available studies, a potential risk of serious adverse events cannot be completely excluded when LABA is used as an add-on to ICS in patients with asthma. It is hoped that the ongoing safety studies will provide an answer to this question.

In contrast to $\beta$-agonists, $\beta$-blockers have for many years been regarded as contraindicated in patients suffering from asthma due to the potential risk of triggering bronchoconstriction, ${ }^{6,7}$ which could potentially result in an insufficient response to bronchodilator therapy during a severe asthma attack. Although not as common as in patients with chronic obstructive pulmonary disease (COPD), especially elderly patients with asthma who may have comorbidities such as chronic heart failure and angina, use of $\beta$-blockers is known to have symptomatic effect and to improve the outcome. ${ }^{8}$ Few studies have examined the risk-benefit ratio of maintenance therapy with $\beta$-blockers in patients with asthma, ${ }^{9}$ and the present contraindication is, therefore, based solely on the risk for bronchoconstriction after acute administration. There have been reports of severe attacks of asthma triggered by a single dose of $\beta$-blockers. ${ }^{10,11}$ However, some researchers have, in line with the positive effect of $\beta$-blockers in patients with left ventricular failure, suggested that $\beta$-blockers may also have a beneficial effect in asthma. ${ }^{6}$ This suggestion has probably at least partly been based on the observations of potential serious adverse effects of LABA therapy for patients with asthma. ${ }^{4,5}$

The density of $\beta$-receptors in airway smooth muscle does not change at different airway levels, so bronchioles have a similar density to large airways, but $\beta$-receptors are also expressed on other potentially important target cells, including airway epithelium, mast cells, type 2 pneumocytes, submucosal glands, and postcapillary venules. Furthermore, inflammatory cells, including eosinophils and neutrophils, also express $\beta$-receptors, which are rapidly desensitized by $\beta$-agonists. The effects of $\beta$-blockers in asthma, whether beneficial or detrimental, may therefore be mediated through several potential pathways.

The aim of this short review is to provide the reader with an overview of our current knowledge of the potential risks and beneficial effects of $\beta$-blockers in the management of asthma.

\section{Methods}

A series of searches, last updated in May 2013, was carried out using the PubMed database. The search strategy was intended to be broad, in order to maximize the capture of citations for peer-reviewed publications relevant to asthma and $\beta$-blockers. The PubMed searches were carried out using the following algorithm of MeSH terms: asthma, asthmalike symptoms and $\beta$-blockers or $\beta$-blockade; the searches were repeated with these terms in combination with reactive airways disease and reversible airway disease. The citation pool was further supplemented from manual assessment of the reference lists accompanying original research and other systematic reviews of aspects related to asthma and $\beta$-blockers and from other publications identified as being relevant for further review. Only publications in English published after 1985 reporting original research (ie, animal models or studies of humans) assessing potential risks and benefits of $\beta$-blocker therapy in asthma were included in this review. Studies evaluating the effect of $\beta$-blocker therapy in patients with COPD only were excluded, primarily because the risk-benefit ratio of $\beta$-blocker therapy differs substantially between patients with asthma and patients with COPD due to differences in the prevalence of comorbidities, including cardiovascular disease.

\section{Results}

\section{The potential risks associated with administration of $\beta$-blockers to asthma patients}

In an observational study, Morales et $\mathrm{al}^{7}$ assessed the possible association between prescription of $\beta$-blockers to asthma patients and the incidence of severe asthma exacerbations requiring treatment with oral corticosteroids. The primary aim was to determine whether the first $\beta$-blocker prescription was associated with a subsequent prescription of a rescue course of oral corticosteroids. Clinical data were obtained from one third of all Scottish general practices, with 1.76 million registered patients. Patients with asthma were identified on March 31, 2007 and data on all asthma-related medication within the preceding 2 years were recorded. Data on all patients with a read code (hierarchical clinical coding system used in the UK; also necessary for reimbursement claims) for asthma as well as patients aged 18-40 years were extracted. An asthma case was defined as an individual with the combination of asthma READ code and no READ code for COPD. In order 
to avoid inclusion of individuals with undiagnosed COPD, the researchers only included patients aged $>40$ years if they were registered as "never smokers." A total of 53,944 adults with asthma were identified, of whom 1,527 patients had had a prescription for a $\beta$-blocker (both nonselective and cardioselective $\beta$-blockers). The cohort of interest comprised the 695 patients receiving the first $\beta$-blocker prescription during the study period. A new oral $\beta$-blocker prescription was defined as the first $\beta$-blocker prescription with a preexposure period of $\geq 84$ days. Active asthma was defined as prescriptions of asthma-related medications, including SABA, LABA, ICS, fixed-combination inhalers (LABA/ICS), theophylline, and leukotriene antagonists, filled between January 1, 2005 and the date of the new $\beta$-blocker prescription. Patients were required to have a follow-up period of at least 84 days to determine the potential effects of $\beta$-blocker therapy on incidence of prescribed rescue courses of oral corticosteroid. Patients receiving nonselective $\beta$-blockers were younger ( $<40$ years of age) and more likely to be women. Patients prescribed selective $\beta$-blockers were more likely to have congestive heart failure and ischemic heart disease. For the 599 patients receiving a new $\beta$-blocker prescription, asthma severity was defined according to the British Thoracic Society guidelines. ${ }^{12}$ Of these 599 patients, 376 (62.8\%) were prescribed SABA, 274 (45.7\%) were prescribed ICS, and $70(11.7 \%)$ were prescribed LABA/ICS prior to the first $\beta$-blocker prescription. Of 441 patients identified as having active asthma, 367 had the required follow-up period and were therefore included in the final analysis. The use of rescue steroids was quantified at baseline, for weeks $0-2$, for weeks 2-4, and for weeks 4-8. The final analysis revealed no statistical difference in the proportion of patients prescribed oral steroids in the weeks following the first $\beta$-blocker prescription. The authors concluded that no large increase was observed in number of patients requiring oral corticosteroids for exacerbation of asthma during the first, possibly most critical, period after the initiation of $\beta$-blocker treatment, although a small increase in risk could not be excluded.

Van Zyl et $\mathrm{al}^{13}$ studied the effects of two cardioselective $\beta$-blockers, ie, celiprolol and atenolol, on respiratory function and asthma control in patients with asthma and concomitant mild to moderate essential hypertension. In contrast to atenolol, celiprolol is claimed to have bronchodilator properties. Patients were eligible for the study provided they had a forced expiratory volume in 1 second $\left(\mathrm{FEV}_{1}\right)<85 \%$ predicted combined with either a $15 \%$ increase in $\mathrm{FEV}_{1}$ in response to salbutamol or a positive histamine challenge test (defined as a $\mathrm{PC}_{20}$ [the provocative dose causing a $20 \%$ fall in $\left.\mathrm{FEV}_{1}\right]$ histamine $<8 \mathrm{mg} / \mathrm{mL}$ ). After a single-blind 2-week run-in period, the enrolled patients $(n=12)$ were randomized in a double-blind placebo-controlled design to either $100 \mathrm{mg}$ atenolol or $400 \mathrm{mg}$ celiprolol daily or vice versa for 4 weeks, with a 2-week washout period in between. Spirometry was performed prior to the first dose of trial medication (celiprolol, atenolol, or placebo), and postdose spirometry was done at the following time points: 30 minutes, 1 hour, 2 hours, and 3 hours. At 3 hours, all patients were given a single dose of inhaled salbutamol, and spirometry was repeated after 5-15 minutes. Patients recorded symptom scores and use of inhaler medication throughout the study period. Ten of 12 patients completed the study; one patient had an acute exacerbation during the study period and one patient required add-on antihypertensive therapy. A progressive fall in $\mathrm{FEV}_{1}$ and forced vital capacity was observed during the 3 hours after the single-dose challenge with atenolol, followed by an improvement to prechallenge levels after administration of salbutamol. In contrast to this, both $\mathrm{FEV}_{1}$ and forced vital capacity remained unchanged after single-dose administration of celiprolol, whereas the response to salbutamol was preserved. The single-dose response after 2 weeks of maintenance $\beta$-blocker therapy was similar to the response observed after placebo. No effects of $\beta$-blocker therapy were observed on symptom scores or use of rescue bronchodilator. On the basis of the difference in response to the two bronchodilators, the authors concluded celiprolol may have a more favorable safety profile than atenolol in patients with asthma. Although this study revealed a $\beta$-blocker-associated fall in lung function and no effect of maintenance therapy on asthma control, it should be noted that information on mean level of lung function and smoking habits was not provided. Inclusion of patients with COPD who met the inclusion criteria primarily due to a low baseline level of lung function is, therefore, a possibility, which may compromise the interpretation of the findings. Similarly, Yamakage et $\mathrm{al}^{14}$ included patients (mean age 59.8 years) with at least two coronary risk factors, airway resistance $>180 \%$ predicted and $\mathrm{FEV}_{1}<70 \%$ predicted, in a study investigating the effects of esmolol and landiolol on wheezing during induction of anesthesia.

In a double-blind, randomized, crossover study, Wilcox et $\mathrm{al}^{15}$ investigated the effect of metoprolol and bevantolol in 16 patients with asthma. Cumulative doses, ie, $12.5 \mathrm{mg}, 25 \mathrm{mg}, 50 \mathrm{mg}$, and $100 \mathrm{mg}$ of metoprolol or $18.75 \mathrm{mg}, 37.5 \mathrm{mg}, 75 \mathrm{mg}$, and $150 \mathrm{mg}$ of bevantolol, were administered at 2-hour intervals. Symptoms and lung function were monitored, and treatment was stopped if significant symptoms or a $20 \%$ decline in $\mathrm{FEV}_{1}$ were observed. 
The cumulative dosing regimen in general proved to be a safe and effective means of assessing bronchial responsiveness to $\beta$-blockers in asthma, but one patient had to be withdrawn after the first dose due to severe bronchoconstriction. Of the 15 patients exposed to both $\beta$-blockers, seven patients were withdrawn prematurely. The maximum tolerated cumulative dose of metoprolol and bevantolol was $26.8 \mathrm{mg}$ and $45.5 \mathrm{mg}$, respectively, doses much lower than usually required for therapeutic activity. The authors concluded that even in patients who tolerate single doses of $\beta$-blockers, the response to repeated treatment is unpredictable and, therefore, that $\beta$-blocker therapy should be avoided in patients with asthma.

\section{Potential benefits of $\beta$-blockers for patients with asthma}

\section{Animal studies}

Observations by Callaerts-Vegh et $\mathrm{al}^{16}$ from a murine model of asthma have shown that, although acute administration of $\beta$-blockers increased the level of airway hyperresponsiveness, longer-term administration (28 days) resulted in a decrease in airway hyperresponsiveness. Furthermore, this study also showed that chronic therapy reduced the total cell count in bronchoalveolar lavage (BAL) fluid, BAL eosinophil counts, and BAL cytokine levels, including interleukin (IL)-5, IL-10, and IL-13. Treatment with a $\beta$-blocker for 28 days also decreased mucin content and partially reversed the pathological changes in the airway epithelium. ${ }^{17,18}$

\section{Human studies}

Bauer et $\mathrm{al}^{19}$ studied the effects of an oral osmotic formulation of metoprolol and atenolol on skeletal muscles and bronchial smooth muscles. They included 28 patients with stable asthma and concomitant essential hypertension, but no other comorbidities. Patients were included if they had a $\mathrm{FEV}_{1}>50 \%$ predicted, an increase in $\mathrm{FEV}_{1}>15 \%$ after administration of $400 \mu \mathrm{g}$ salbutamol, and a diastolic blood pressure $>90 \mathrm{mmHg}$. The electrocardiogram and chest X-ray were normal in all patients. The study was a randomized, double-blind, three-period, crossover, placebocontrolled trial with at least a 7-day washout phase between treatment periods. The study drugs were administered once daily in all treatment periods. On treatment days 1 and 7, $\mathrm{FEV}_{1}$, specific airway conductance, finger tremor, blood pressure, and heart rate were measured. Dose-response curves were then constructed using six increasing doses of inhaled salbutamol, as follows: $12.5 \mu \mathrm{g}, 25 \mu \mathrm{g}, 75 \mu \mathrm{g}$, $300 \mu \mathrm{g}, 400 \mu \mathrm{g}$, and $800 \mu \mathrm{g}$, dose increments were made at 20-minute intervals, and a 5-minute period was scheduled for recording all parameters. A total of 18 patients completed the study and were included in the analyses. Ten patients were excluded: six withdrew for personal reasons, one had an upper respiratory tract infection, one experienced finger tremor and palpitations of moderate degree during the salbutamol dose response challenge while on placebo therapy (day 1) and declined to continue, and two experienced a worsening of their asthma (one patient after having finished the placebo and metoprolol treatment period and one after the metoprolol and atenolol phase). No statistically significant differences in baseline characteristics were found between the ten patients excluded and the 18 patients who completed the study. The study showed that a single dose (day 1) of both metoprolol and atenolol had no measurable influence on bronchial $\beta_{2}$-adrenergic receptors as assessed by the salbutamol dose-response curve in hypertensive asthma patients. Neither metoprolol nor atenolol caused a difference in postmedication (after 30 minutes of rest) baseline lung function before administration of salbutamol, and the salbutamol dose-response curves obtained after active treatment were indistinguishable from those obtained after placebo. Multiple doses of metoprolol caused no measurable bronchial $\beta_{2}$-adrenergic receptor antagonism in the patients, whereas multiple doses of atenolol caused a significant shift to the right in the salbutamol dose-response curve. No significant influence was reported on bronchomotor tone in hypertensive asthma patients when treated with either single or multiple doses of metoprolol, although multiple dosing did cause blockade on $\beta_{2}$-adrenergic receptors of skeletal muscle. The authors concluded that metoprolol had no measurable influence on bronchomotor tone; nonetheless, other side effects and risks could arise when $\beta$-blockers are administered to asthma patients.

In a pilot study, Hanania et $\mathrm{al}^{3}$ recruited ten steroid-naïve patients with asthma into a prospective open-label, doseescalating study investigating the safety of the nonselective $\beta$-blocker nadolol. All participants fulfilled the following criteria: (1) diagnosed with asthma, (2) aged 18-50 years, (3) nonsmokers or past smokers with a history of $<10$ pack years, (4) baseline prebronchodilator $\mathrm{FEV}_{1} \geq 80 \%$ of predicted value, $\mathrm{PC}_{20}<8 \mathrm{mg} / \mathrm{mL}$ methacholine, and (6) baseline blood pressure $\geq 110 / 70 \mathrm{mmHg}$ and pulse rate $\geq 60$ beats per minute. Patients were not eligible for the study if they had other significant health issues and/or had been using any controller medication for asthma within 4 weeks of baseline. The patients were followed for 11 weeks and received medication (nadolol) for 9 weeks. After a 2-week run-in period, eligible patients were 
entered into a dose-escalating phase lasting up to 6 weeks and then into a 3 -week period on stable dose. The initial dose was $10 \mathrm{mg}$ once daily and it was escalated, maintained, or reduced every week at the study visits on the basis of predetermined criteria, including level of lung function. All participants completed the study and tolerated a maximum nadolol dose of $10 \mathrm{mg}(\mathrm{n}=3), 20 \mathrm{mg}(\mathrm{n}=4)$, or $40 \mathrm{mg}(\mathrm{n}=3)$. The study showed a dose-dependent improvement in $\mathrm{PC}_{20}$ methacholine in eight of ten patients and a 5\%, statistically significant, decrease in $\mathrm{FEV}_{1}$. The decline in $\mathrm{FEV}_{1}$ was asymptomatic, and there was no significant correlation between the maximum dose of nadolol tolerated and the fall in $\mathrm{FEV}_{1}$. The study indicates that treatment of asthma patients with a nonselective $\beta$-blocker, nadolol, is well-tolerated and actually may have a beneficial effect when repeated daily doses are administered in patients with mild asthma not on controller medication.

Hanania et $\mathrm{al}^{20}$ completed another open-label study of chronic treatment with nadolol, also in patients with mild asthma not on controller therapy. The study comprised ten patients, used a dose-escalating model, and was 13 weeks long, including at least three weeks on the final tolerated dose. The initial dose of $1.25 \mathrm{mg}$ was escalated biweekly depending on predetermined criteria, ie, lung function, asthma control, and hemodynamic parameters. Seven of the ten patients tolerated a maximum dose of $40 \mathrm{mg}$; one tolerated a daily dose of $10 \mathrm{mg}$ and one a daily dose of $5 \mathrm{mg}$. One participant was excluded from the study because of an asthma exacerbation while being treated with the minimal dose of $1.25 \mathrm{mg}$. Results indicated a significant effect on airway hyperresponsiveness and no significant changes in $\mathrm{FEV}_{1}$. In participants from both studies by Hanania et $\mathrm{al}^{3,20}(\mathrm{n}=18)$, bronchodilator responsiveness to salbutamol was evaluated immediately after the methacholine challenge test at the final visit. Spirometry performed 20 minutes after administration of salbutamol showed that salbutamol reversed the methacholine-induced bronchospasm in all nadolol-treated patients. This is similar to observations in patients with asthma and no history of treatment with $\beta$-blockers, for whom administration of salbutamol after methacholine challenge led to a faster recovery of $\mathrm{FEV}_{1}$ compared with placebo. ${ }^{21}$ The former findings, therefore, suggest that $\beta$-blocker therapy does not inhibit the bronchodilating effect of salbutamol.

In keeping with this, Short et $\mathrm{al}^{22}$ investigated the safety of acute exposure to propranolol in patients with asthma challenged with histamine in order to mimic an asthma exacerbation, and observed that nebulized salbutamol and ipratropium bromide produced a full recovery of bronchoconstriction induced by propranolol and histamine.
In a very recent double-blind, placebo-controlled crossover study, Short et $\mathrm{al}^{23}$ assessed the effect of the nonselective $\beta$-blocker propranolol as add-on to ICS in adults with asthma. A total of 18 patients classified as having mild to moderate asthma based on level of lung function (mean $\mathrm{FEV}_{1}$ 93\% predicted) and prescribed medium dose of ICS (mean daily dose $440 \mu \mathrm{g}$ ) completed the study. The study protocol consisted of a 6-8-week dose titration of propranolol or placebo as tolerated by the individual participants, up to a maximum daily dose of $80 \mathrm{mg}$; the primary outcome variable was airway responsiveness to inhaled methacholine. Apart from add-on propranolol or placebo, all participants were also treated with tiotropium bromide once daily for the first 4-6 weeks of each treatment period. The treatment response was also evaluated by histamine responsiveness, lung function, and questionnaires (Mini Asthma Quality Of Life Questionnaire and Asthma Control Questionnaire). No effect of propranolol versus placebo was observed on airway responsiveness to methacholine $(P=0.89)$, and likewise no difference was reported in histamine responsiveness or responses to the questionnaires. However, a small but statistically significant decrease in salbutamol responsiveness after the histamine challenge test was observed (mean difference in $\mathrm{FEV}_{1} 5.3 \%$ of the predicted value), and a small decline in $\mathrm{FEV}_{1}$ was also observed at the end of the propranolol treatment period. Apart from not supporting the concept of $\beta$-blocker therapy in asthma, this small negative controlled trial may have important implications for possible future trials in relation to the target population, duration of therapy, and concurrent treatment with longacting bronchodilators.

\section{Discussion}

Initiation of $\beta$-blocker treatment for diseases other than asthma is not associated with an increase in the need for rescue courses of oral corticosteroid for an exacerbation of asthma. ${ }^{7}$ However, patients with mild to moderate reactive airway disease, in most studies likely including both asthma and COPD, may have a limited decrease in $\mathrm{FEV}_{1}$ after single-dose administration of $\beta$-blocker, whereas no change in $\mathrm{FEV}_{1}$ has been reported after long-term administration. ${ }^{9}$ Also reassuring is the observation by Hanania et $\mathrm{al}^{3,20}$ that asthma patients treated with $\beta$-blockers have a preserved and sufficient response to bronchodilators. Even though a potential risk cannot be ruled out and the intraindividual variation should be remembered, the available studies suggest that chronic use of cardioselective $\beta$-blockers, at least, is well-tolerated in asthma patients. 
On the other hand, evidence from both human and animal studies has revealed positive effects on airway hyperresponsiveness and a probable anti-inflammatory effect with the chronic use of $\beta$-blockers. ${ }^{3,16,20}$ However, the very recent randomized placebo-controlled study by Short et $\mathrm{al}^{23}$ revealed no positive effect of treatment with a nonselective $\beta$-blocker on airway responsiveness to methacholine or histamine, lung function, and symptoms in patients with mild to moderate asthma (mean $\mathrm{FEV}_{1}$ 93\% predicted) treated with mediumdose ICS. The study by Short et al, ${ }^{23}$ although not lending support to the concept of a positive effect of $\beta$-blockers in asthma, may suggest that future controlled trials addressing this question should perhaps include patients with more severe asthma. This would be in keeping with the reported positive effects of $\beta$-blocker therapy for patients with heart failure. It is well documented that obese individuals not only have a high prevalence of asthma, but also have less-favorable response to current recommended asthma therapy. ${ }^{24}$ One of the possible mechanisms underlying the association between obesity and asthma may be increased stiffness of airway smooth muscle, ${ }^{25}$ and obese patients with asthma might, therefore, also be a target group in future trials of $\beta$-blocker therapy for asthma. Furthermore, in the study by Short et al, ${ }^{23}$ patients were treated with add-on tiotropium bromide for most of the study period. Recent studies in patients with asthma suggest that tiotropium may have important anti-inflammatory effects, ${ }^{26}$ and adding tiotropium to the treatment regimen in the study by Short et $\mathrm{al}^{23}$ may, therefore, have blunted the effect of the primary intervention.

The observations in currently available studies, although limited, clearly suggest that large-scale clinical trials, probably using a dose-escalating model, are needed in order to explore the potential positive effect of chronic treatment with $\beta$-blockers in patients with asthma, including patients with more severe asthma.

\section{Disclosure}

The authors report no conflicts of interest in this work.

\section{References}

1. Global Initiative for Asthma [homepage on the Internet]. Global Initiative for Asthma; 2013. Available from: http://www.ginasthma.org. Accessed June 15, 2013.

2. Grove A, Lipworth BJ. Bronchodilator subsensitivity to salbutamol after twice daily salmeterol in asthmatic patients. Lancet. 1995;346(8969): 201-206.

3. Hanania NA, Singh S, El-Wali R, et al. The safety and effects of the beta-blocker, nadolol, in mild asthma: an open-label pilot study. Pulm Pharmacol Ther. 2008;21(1):134-141.
4. Nelson HS, Weiss ST, Bleecker ER, Yancey SW, Dorinsky PM; for the SMART Study Group. The Salmeterol Multicenter Asthma Research Trial: a comparison of usual pharmacotherapy for asthma or usual pharmacotherapy plus salmeterol. Chest. 2006;129(1):15-26.

5. Castle W, Fuller R, Hall J, Palmer J. Serevent nationwide surveillance study: comparison of salmeterol with salbutamol in asthmatic patients who require regular bronchodilator treatment. $B M J$. 1993;306(6884):1034-1037.

6. Dickey BF, Walker JK, Hanania NA, Bond RA. beta-Adrenoceptor inverse agonists in asthma. Curr Opin Pharmacol. 2010;10(3): 254-259.

7. Morales DR, Guthrie B, Lipworth BJ, Donnan PT, Jackson C. Prescribing of $\beta$-adrenoceptor antagonists in asthma: an observational study. Thorax. 2011;66(6):502-507.

8. Lechat P, Packer M, Chalon S, Cucherat M, Arab T, Boissel JP. Clinical effects of beta-adrenergic blockade in chronic heart failure: a meta-analysis of double-blind, placebo-controlled, randomized trials. Circulation. 1998;98(12):1184-1191.

9. Salpeter S, Ormiston T, Salpeter E. Cardioselective beta-blockers for reversible airway disease. Cochrane Database Syst Rev. 2002;(4): CD002992.

10. Raine JM, Palazzo MG, Kerr JH, Sleight P. Near-fatal bronchospasm after oral nadolol in a young asthmatic and response to ventilation with halothane. Br Med J (Clin Res Ed). 1981;282(6263):548-549.

11. Spitz DJ. An unusual death in an asthmatic patient. Am J Forensic Med Pathol. 2003;24(3):271-272.

12. British Thoracic Society. Guidelines [webpage on the Internet]. London, UK: British Thoracic Society; 2013. Available from: http://www. brit-thoracic.org.uk/guidelines/asthma-guidelines.aspx. Accessed May 2013.

13. van Zyl AI, Jennings AA, Bateman ED, Opie LH. Comparison of respiratory effects of two cardioselective beta-blockers, celiprolol and atenolol, in asthmatics with mild to moderate hypertension. Chest. 1989;95(1):209-213.

14. Yamakage M, Iwasaki S, Jeong SW, Satoh J, Namiki A. Beta-1 selective adrenergic antagonist landiolol and esmolol can be safely used in patients with airway hyperreactivity. Heart Lung. 2009;38(1):48-55.

15. Wilcox PG, Ahmad D, Darke AC, Parsons J, Carruthers SG. Respiratory and cardiac effects of metoprolol and bevantolol in patients with asthma. Clin Pharmacol Ther. 1986;39(1):29-34.

16. Callaerts-Vegh Z, Evans KL, Dudekula N, et al. Effects of acute and chronic administration of beta-adrenoceptor ligands on airway function in a murine model of asthma. Proc Natl Acad Sci U SA. 2004;101(14): 4948-4953.

17. Lin R, Peng H, Nguyen LP, et al. Changes in beta 2-adrenoceptor and other signaling proteins produced by chronic administration of 'betablockers' in a murine asthma model. Pulm Pharmacol Ther. 2008;21(1): $115-124$.

18. Nguyen LP, Omoluabi O, Parra S, et al. Chronic exposure to betablockers attenuates inflammation and mucin content in a murine asthma model. Am J Respir Cell Mol Biol. 2008;38(3):256-262.

19. Bauer K, Kaik G, Kaik B. Osmotic release oral drug delivery system of metoprolol in hypertensive asthmatic patients. Pharmacodynamic effects on beta 2-adrenergic receptors. Hypertension. 1994;24(3):339-346.

20. Hanania NA, Mannava B, Franklin AE, et al. Response to salbutamol in patients with mild asthma treated with nadolol. Eur Respir $J$. 2010;36(4):963-965.

21. Politiek MJ, Boorsma M, Aalbers R. Comparison of formoterol, salbutamol and salmeterol in methacholine-induced severe bronchoconstriction. Eur Respir J. 1999;13(5):988-992.

22. Short PM, Williamson PA, Lipworth BJ. Effects of hydrocortisone on acute $\beta$-adrenoceptor blocker and histamine induced bronchoconstriction. Br J Clin Pharmacol. 2012;73(5):717-726.

23. Short PM, Williamson PA, Anderson WJ, Lipworth BJ. Randomised placebo controlled trial to evaluate chronic dosing effects of propranolol in asthma. Am J Respir Crit Care Med. Epub April 17, 2013. 
24. Juel CT, Ulrik CS. Obesity and asthma: impact on severity, asthma control, and response to therapy. Respir Care. 2013;58(5):867-873.

25. Ali Z, Ulrik CS. Obesity and asthma: a coincidence or a causal relationship? A systematic review. Respir Med. Epub May 1, 2013.
26. Antoniu SA, Antohe I. Evaluation of inhaled tiotropium in asthma, uncontrolled with standard combination therapy. Expert Opin Pharmacother. 2013;14(7):967-969.

\section{Publish your work in this journal}

The International Journal of General Medicine is an international, peer-reviewed open-access journal that focuses on general and internal medicine, pathogenesis, epidemiology, diagnosis, monitoring and treatment protocols. The journal is characterized by the rapid reporting of reviews, original research and clinical studies across all disease areas.
A key focus is the elucidation of disease processes and management protocols resulting in improved outcomes for the patient.The manuscript management system is completely online and includes a very quick and fair peer-review system. Visit http://www.dovepress.com/ testimonials.php to read real quotes from published authors.

Submit your manuscript here: http://www.dovepress.com/international-journal-of-general-medicine-journal 